\title{
Application of acupuncture in the treatment of venous insufficiency and varicose veins
}

\author{
Hayriye Alp (D) \\ GETAT Center, Necmettin Erbakan University Meram Medical Faculty Hospital, Konya, Turkey
}

Received: May 03, 2018 Accepted: May 03, 2018 Published online: March 20, 2020

\begin{abstract}
A 43-year-old male patient was admitted with swollen legs, which was prominent in the right leg. He was under treatment for varicose veins due to femoral and saphenous insufficiency for 10 years. Edema, redness, and cyanosis were present in his toes on physical examination. According to the pulse examination, organ meridians with reduced energy were applied to the body acupuncture needles (Liv-3, St-41, Kid-6, $\mathrm{St}-25,24, \mathrm{Sp}-9)$. The ear was stabbed in the ankle, Shen men's point. Diet for weight control of the leg was suggested. Thanks to the most effective acupuncture points for edema resolution, the measurements yielded a $4-\mathrm{cm}$ decrease in the tibia plateau and 3-cm decrease in the ankle circumference. The patient's pain and cyanosis decreased. Local acupuncture was applied around the contracture as the patient's complaints diminished. In conclusion, acupuncture as a holistic approach can be a useful method in the treatment of venous insufficiency and varicose veins.

Keywords: Acupuncture, edema, venous insufficiency.
\end{abstract}

Acupuncture is a therapy of healing the body in which the fine needles are inserted at specific locations on the body. ${ }^{[1]}$ It is mostly used in pain relief in Turkey. Although there are more than 350 acupuncture points lying along the meridians, yuan source points, sedation points, and horary points are the most commonly used points. ${ }^{[2]}$ Acupuncture is believed to activate the endogenous opioid system and produces analgesic effects and stimulate parasympathetic system by oxytocin release. ${ }^{[3]}$ Acupuncture is not only a discipline, but also it is used in the treatment of several diseases. There are 12 principal meridians and extra meridians in the human body. Meridians are mapped similar to the meridians of the geographical globe. The starting and end points of the meridians are located on the fingertips and toes. All meridians are connected to a network known as primo vascular system and regulate the interaction of cells with the connective tissues. ${ }^{[4]}$ The acupuncture points are located on the line arm meridians over the body surface. These points can be stimulated by needle, push, ultrasound, light, or electrical currents. ${ }^{[3]}$

Herein, we report a male case of venous insufficiency and varicose veins which were successfully relieved with acupuncture.

\section{CASE REPORT}

A 43-year-old male patient was admitted with swollen legs, which was prominent in the right leg.
He was under treatment for varicose veins due to femoral and saphenous insufficiency for 10 years. Edema, redness, and cyanosis were present in his toes on physical examination. According to the pulse examination, organ meridians with reduced energy were applied to the body acupuncture needles (Liv-3, St-41, Kid-6, St-25,24, Sp-9). The ear was stabbed in the ankle, Shen men's point. Diet for weight control of the leg was suggested. The patient's pain and cyanosis decreased. Local acupuncture was applied around the contracture as the patient's complaints diminished. A written informed consent was obtained from the patient.

\section{DISCUSSION}

Specific nerves are stimulated, when specific points on the body are stimulated. ${ }^{[3]}$ By means of the neurovegetative system, the peripheral parts of the body are controlled in direct or indirect relation with it segments by the medulla spinalis. The stimulation

Corresponding author: Hayriye Alp, MD. Necmettin Erbakan Üniversitesi Meram Tip Fakültesi Hastanesi GETAT Merkezi, 42080 Meram, Konya, Türkiye. Tel: +90 332 - 2237312 e-mail: hayriyebalp@yahoo.com

Citation:
Alp H. Application of acupuncture in the treatment of venous insufficiency and
varicose veins. Cardiovasc Surg Int 2019;7(2):31-33.


of a nerve from this group affects other parts of the body. This effect can be sometimes an increase or decrease in activity. Increased activity by stimulation of the sympathetic nerves becomes reduced through the stimulation of the parasympathetic nerves. ${ }^{[1,2]}$ An increased heart rate, decreased blood vessels, tightening and loosening of the blood vessels, and increased or decreased hormone secretion may be due to the same mechanism. Acupuncture provides a significant improvement in the treatment of venous ulcers. ${ }^{[5,6]}$ According to the traditional Chinese medicine, edema travelling from the knee toward the lower limb indicates spleen and kidney Yang deficiency with the accumulation and retention of damp and mucus, thereby, leading to edema. ${ }^{[7]}$ Using the acupuncture technique, meridians of the spleen and kidney are the location for edema resolution. In a study, intradermal needling of the Sanyinjiao (SP-6) and Fliu (KI-7) intersecting points diminished the structures around the limb. ${ }^{[8]}$

In the published Yellow Emperor's Internal Classic, lung and colon meridians should be used in the treatment of upper back-related diseases and stomach and spleen meridians in the treatment of low back-related diseases. ${ }^{[9]}$ ST-36 is an acupoint of the stomach meridian and rich in $\mathrm{Qi}$ and blood and its stimulation is thought to regulate all body functions. ${ }^{[10]}$ In addition, acupuncture is effective in the treatment of edema due to damp and mucus. The SP-6 is the intersecting point of kidney, liver, and spleen meridians and regulates vital Qi flow, enhancing the kidney and spleen energy. ${ }^{[1]}$ In a study, moxibustion with a warming needle was found to be more effective in edema resolution than acupuncture alone. ${ }^{[11]}$ There are also studies showing the efficacy of acupuncture in reducing the number of migraine attacks. ${ }^{[12,13]}$ Some authors also reported that acupuncture accelerated healing process, resolved edema, and provided analgesia in a case of metatarsal fracture. ${ }^{[14]}$

In conclusion, to the best of our knowledge, this is the first case report to show the effectiveness of acupuncture in the treatment of venous insufficiency and varicose veins. Currently, pharmacological and surgical approaches are commonly used, and our findings are required to be reproduced in further studies. We believe that acupuncture as an adjunct method to conventional approaches is helpful to improve the quality of life of patients.

\section{Declaration of conflicting interests}

The author declared no conflicts of interest with respect to the authorship and/or publication of this article.

\section{Funding}

The author received no financial support for the research and/or authorship of this article.

\section{REFERENCES}

1. Çevik C. Medical Acupuncture. 3rd ed. Ankara: Kuban Publishing; 2016.

2. Liu, G.W. Acupuncturology. Shanghai: Oriental Medicine Publishing House; 2000. p. 60-2.

3. Zhou W, Benharash P. Effects and mechanisms of acupuncture based on the principle of meridians. J Acupunct Meridian Stud 2014;7:190-3.

4. Stefanov M, Stoev S, Kim J, Kim S. Western Medicine Versus Eastern Medicine: Do Both Have a Common Root, Scientific Background, and Worldwide Recognition? Altern Ther Health Med 2019 Jun 1. pii: AT5744. [Epub ahead of print]

5. Li A, Zhang RX, Wang Y, Zhang H, Ren K, Berman $\mathrm{BM}$, et al. Corticosterone mediates electroacupunctureproduced anti-edema in a rat model of inflammation. BMC Complement Altern Med 2007;7:27.

6. Białoszewski D, Woźniak W, Zarek S. Clinical efficacy of kinesiology taping in reducing edema of the lower limbs in patients treated with the ilizarov method-preliminary report. Ortop Traumatol Rehabil 2009;11:46-54.

7. Long Q, Li J, Wen Y. Clinical observation of auricular point sticking combined with western medicine for preventing and treating postoperative complications of external excision and internal ligation on mixed hemorrhoid. Zhongguo Zhen Jiu 2015;35:237-40.

8. Sumimoto EM, Nishimori AR, Watanabe SN. Effects of press tack needle to edema. J Japan CollAssoc Orient Med 2013;37:35-6. [Abstract]

9. Liu GW, Cao LY. A Complement Work of Present Acupuncture and Moxibustion: Acupoints \& Meridians. 1st ed. Shanghai: Huaxia Publishing House; 1997.

10. Xu H, Zhang Y, Sun H, Chen S, Wang F. Effects of acupuncture at GV20 and ST36 on the expression of matrix metalloproteinase 2, aquaporin 4, and aquaporin 9 in rats subjected to cerebral ischemia/reperfusion injury. PLoS One 2014;9:97488.

11. Zhang WP, Onose Y, Fujikawa T. A Trial Study of Moxibustion with a Warming Needle on Edema. J Acupunct Meridian Stud 2017;10:20-5.

12. Gündüztepe Y, Mit S, Geçioglu E, Gurbuz N, Salkaci O, Severcan C, et al. The impact of acupuncture treatment on nitric oxide (NO) in migraine patients. Acupunct Electrother Res 2014;39:275-83. 
13. Cayir Y, Ozdemir G, Celik M, Aksoy H, Akturk Z, Laloglu E, et al. Acupuncture decreases matrix metalloproteinase-2 activity in patients with migraine. Acupunct Med 2014;32:376-80.
14. Sezgin Y, Becel S, Akçay F. Acupuncture Treatment of a Metatarsus Proximal End Fracture: A Case Report. J Acupunct Meridian Stud 2016;9:319-21. 